\title{
A Novel Composite Piezoelectric Energy Harvester
}

\author{
Qinghua Liu ${ }^{a}$, Zhigang Yang ${ }^{b}$, Yue Wu*c ${ }^{* c}$ Qianjin Tu ${ }^{d}$ and Dongdong Wei ${ }^{\mathrm{e}}$ \\ College of Mechanical Science and Engineering, Jilin University, Changchun 130025, China \\ aliuqh16@mails.jlu.edu.cn, byzg@jlu.edu.cn, \\ cwuyue@jlu.edu.cn, ${ }^{\mathrm{c}}$ tuqj14@mails.jlu.edu.cn, ${ }^{\complement}$ weidd16@mails.jlu.edu.cn
}

Keywords: piezoelectric generator, TPMS, energy harvester, composite structure

Abstract. The passive energy harvesting technique of piezoelectric cantilever is widely researched in TPMS. The energy harvester characteristics of different installation modes are compared, found the energy capture capability is complementary under different installation conditions, therefore a novel composite piezoelectric cantilever energy harvester is designed for TPMS monitoring module, it can increase the bandwidth and self-generating capacity of the device. This structure is effective in the normal speed $(30 \mathrm{~km} / \mathrm{h}-120 \mathrm{~km} / \mathrm{h})$ of the vehicle.

\section{Introduction}

Piezoelectric materials are widely used in environmental energy harvesting. The effective energy harvester is needed with the development of Micro-Electro-Mechanical System (MEMS) [1]. The methods of harvesting energy from the surrounding environment include electromagnetic [2], electrostatic [3] and piezoelectric [4,5]. The piezoelectric harvester has the advantages of no electromagnetic interference, no pollution, easier miniaturization or integration, simpler structure compared with the other two methods. The non-linear energy harvester with centrifugal force [6] , elastic force[7] and electromagnetic force [8,9] can improve the energy harvesting band and improve the energy harvesting efficiency. When the tire rotation was used to produces external excitation, the traditional structure have a narrower operating frequency which is not suitable to supply power for TPMS . When the natural frequency of the device differ from the rotational frequency of the vehicle, the energy harvesting efficiency becomes low. Gao [8] designed a flexible supporting piezoelectric oscillator with the magnet on the top, and.00.bistable transition oscillation was used to improve the energy harvesting efficiency . Eichhorn ect. [10] design a cantilever structure with normal load on the vertical cantilever. The resonant frequency of the cantilever can be reduced from $380 \mathrm{~Hz}$ to $292 \mathrm{~Hz}$, and the energy acquisition efficiency can be improved obviously. Lei [6] studied the natural frequency of cantilever under the action of centrifugal force, which enlarge the resonance frequency. In order to achieve a better energy harvesting efficiency a combined cantilever is designed .

\section{Structure and Principle}

A typical piezoelectric cantilever energy harvester shown in figure 1, which is used in energy capture due to the simple structure. The device comprises a piezoelectric bimorph, a copper substrate, and a top mass block. The piezoelectric cantilever deformed under the inertia effect of mass when the base was activated, then converted into electrical energy.

When the piezoelectric cantilever is arranged in a automobile tires for TPMS, the harvester rotate with the wheel. The base of the cantilever is actuated periodically by the gravity of the top mass. The piezoelectric wafer is pasted onto the surface of one end of the substrate. There are six installation types of cantilever on the wheel hub, however, it is proved that four of them were effective, shown in figure2a-2d. 


\section{Experimental}

The experiment system shown in figure 3 includes a power source, inverter, motor, oscilloscope, safety protection device and rotating plate. The inverter is used to change the rotate speed. The rotating plate is used to fix the piezoelectric cantilever. The piezoelectric wafer is connected to the circuit to measure the voltage of the harvester.

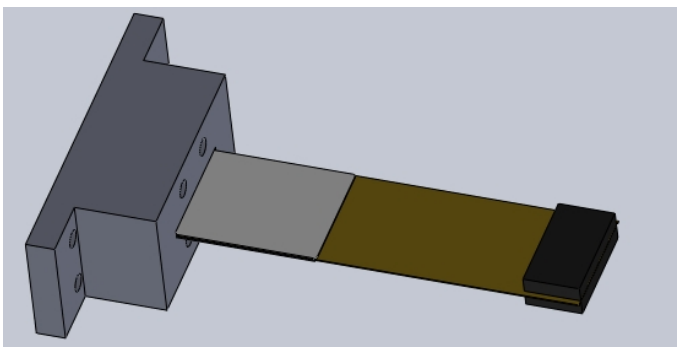

Fig.1 The typical piezoelectric cantilever energy harvester

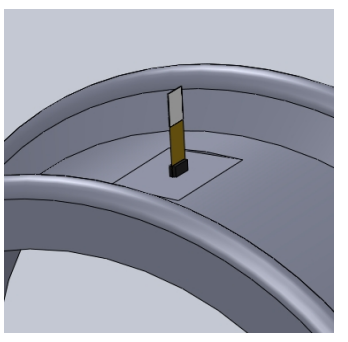

$\mathrm{a}$

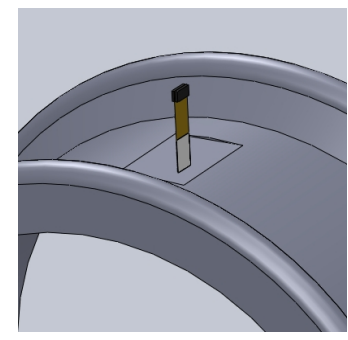

b

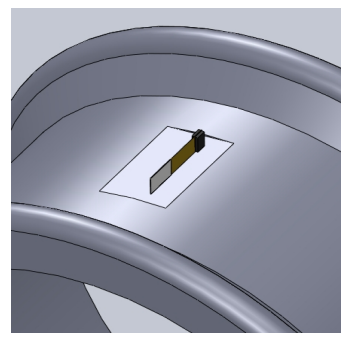

c

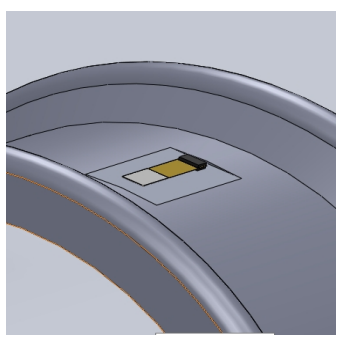

d

Fig.2 Four typical arrangement types

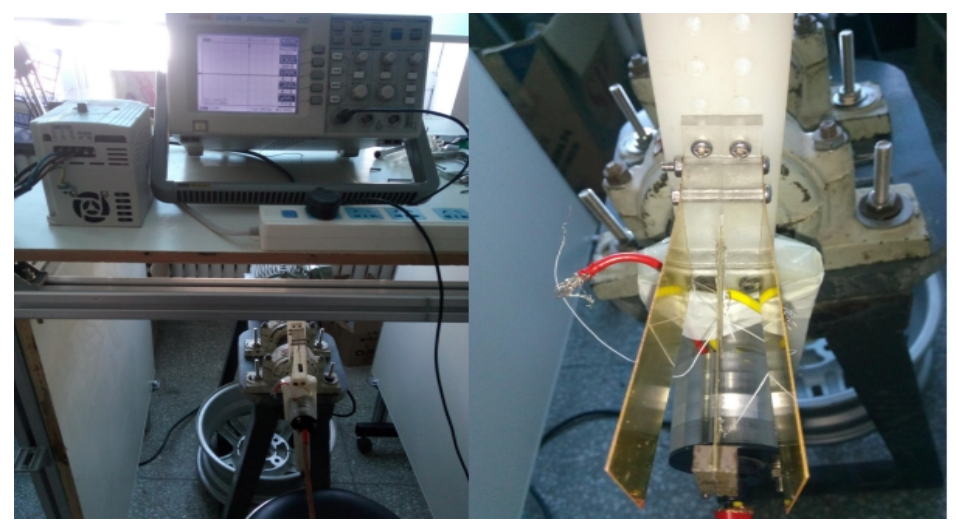

Fig. 3 Experimental Device

The frequency characteristics of different arrangement types of harvesters are shown in figure 4 . Curve 1 represents the voltage of harvester which is shown in figure2a; Curve 2 indicates the voltage of harvester which is shown in figure $2 \mathrm{~b}$; Curve 3 represents the voltage of harvester which is shown in figure2c; Curve 4 represents the voltage of harvester which is shown in figure $2 \mathrm{~d}$.

As can be seen from the diagram, when the plane of the cantilever passes through the center of rotation, as shown in figure $2 \mathrm{a}$, the energy harvesting capability is strengthened between $7 \mathrm{~Hz}-9 \mathrm{~Hz}$, the output voltage is greater than $15 \mathrm{~V}$, which can meet the requirements of TPMS. At resonance frequency $7.5 \mathrm{HZ}$, the voltage reaches $60 \mathrm{~V}$. While the voltage is maintained at $10 \mathrm{~V}$ between $1.5 \mathrm{~Hz}-7 \mathrm{~Hz}$, which cannot meet the requirements of energy supply. When the top mass block deviates from the center of the wheel, as shown in figure $2 \mathrm{~b}$, the energy harvesting voltage is about $15 \mathrm{~V}$ between $1.5 \mathrm{~Hz}-7.5 \mathrm{~Hz}$, which can meet the requirements of energy supply. When the width of the piezoelectric cantilever is pointed to the wheel center, as shown in figure2c, the energy harvesting capability is gradually increased until the resonance is reached, which can meet the requirements of TPMS. When the thickness of the cantilever points to the wheel center, as shown in figure $2 \mathrm{~d}$, the 
voltage of the harvester has no obvious change, and is less than 10V. Therefore, it can be shown that the combination of the first three modes can play a complementary role.

In the experiment, it is found that the centrifugal force is effective to enhance energy harvesting capacity. The voltage is detected respectively at the distance of $77 \mathrm{~mm}, 97 \mathrm{~mm}, 127 \mathrm{~mm}$ between the piezoelectric cantilever and wheel center. The results shown in Figure 5, it can be seen that there is an optimal value to strengthen the capability of the harvester.

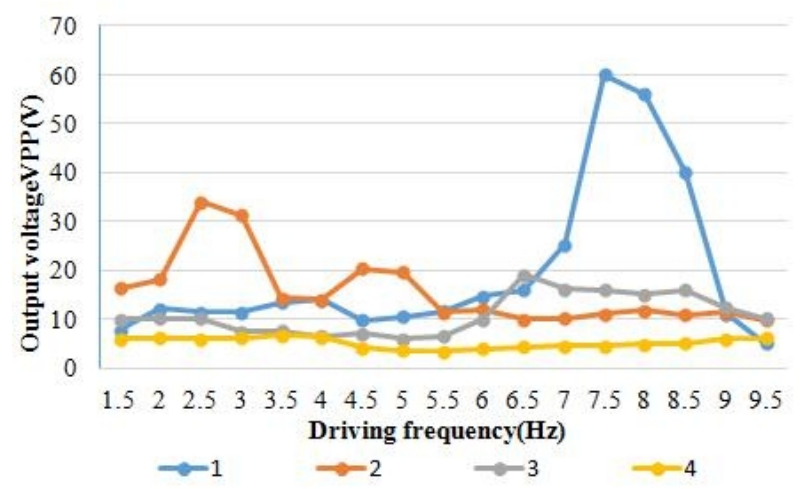

Fig. 4 The frequency characteristics of the four types

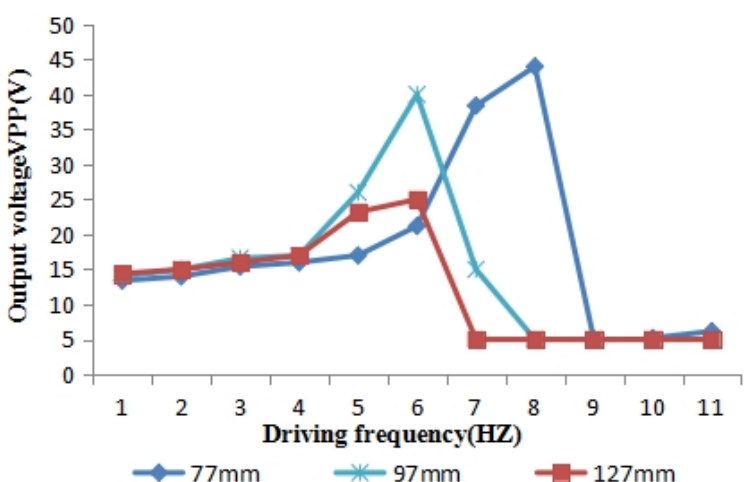

Fig.5 The frequency characteristics under different centrifugal forces

A novel composite piezoelectric cantilever structure was designed using the above results, as shown in Figure 6. It contains three cantilevers, and the arrangement modes are shown in figure 2a, figure $2 b$ and figure $2 c$ respectively. It is mounted on the wheel as shown in Figure 7.

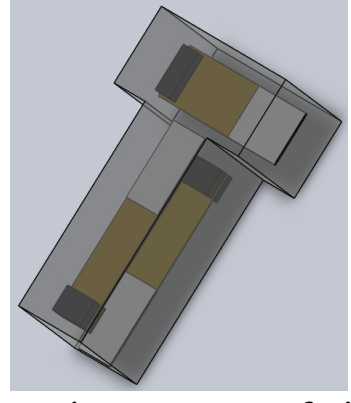

Fig.6 Composite structure of piezoelectric

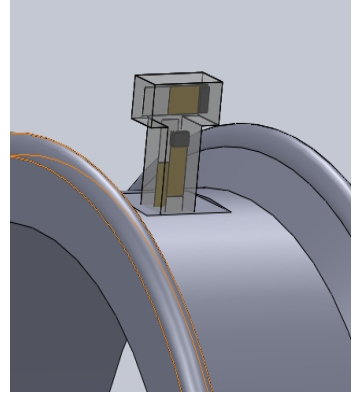

Fig. 7 Installation position

TPMS requires constant DC power at work, but the output voltage of the cantilever is AC. Therefore a standard AC-DC energy harvesting circuit is used in the experiment which is shown in figure 8.The experimental results show that energy storage circuit voltage can be achieved to TPMS power requirements within one minute under the normal driving speed.

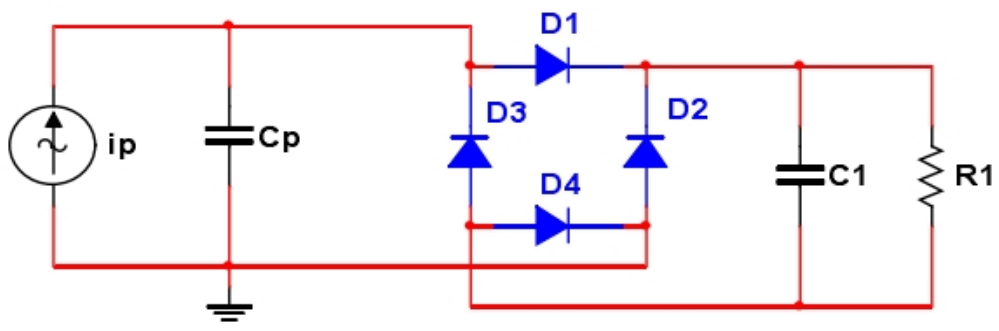

Fig.8 Standard AC-DC energy harvesting circuit 


\section{Conclusion}

This paper compares the characteristics of four different arrangement of piezoelectric cantilever energy harvester, found the centrifugal force along the length direction can reduce the resonant frequency to $3 \mathrm{~Hz}-9 \mathrm{~Hz}$. When the cantilever width or thickness appointing to rotation center, there is a better generation capacity at higher frequencies, but the latter arrangement is proved to be ineffective. A novel composite piezoelectric cantilever energy harvester is designed, which contains three installation modes of cantilever beam and can meet the power requirements for TPMS below the 80 $\mathrm{km} / \mathrm{h}$ driving speed .

\section{Acknowledgements}

This work was financially supported by the National Natural Science Foundation of China (Grant No. 51277088), The corresponding author is Yue Wu.

\section{References}

[1] Y. Wangle and W. Zhu, MEMS Technology and Developing Trend. Mechanical Design \& Research, 2004(01): p. 10-12.

[2] P. Wang, X. Dai, and X. Zhao, A Survey of Microelectromagnetic vibration energy harvesters. Journal of vibration and shock, 2007(09): p. 94-98.

[3] L. Fu, Research on Nonlinear Electrostatic Energy Collector Based on MEMS Technology. 2016.

[4] Kim, S., W.W. Clark, and Q.M. Wang, Piezoelectric Energy Harvesting Using a Clamped Circular Plate: Experimental Study. Journal of Intelligent Material Systems \& Structures, 2005. 16(10): p. 855-863.

[5] Ng, T.H., Sensitivity Analysis and Energy Harvesting for a Self-Powered Piezoelectric Sensor. Journal of Intelligent Material Systems \& Structures, 2005. 16(10): p. 785-797.

[6] Gu, L. and C. Livermore, Passive self-tuning energy harvester for extracting energy from rotational motion. Applied Physics Letters, 2010. 97(8): p. 081904-081904-3.

[7] Y. Wu, J. Dong, X. Li, Z. Yang, Q. Liu, Non-Linear Piezoelectric Actuator with a Preloaded Cantilever Beam, micromachines, 2015. 6(8) p. 1066-1081.

[8] Y. Gao, et al., Studies on vibration resp onse and energy harvesting of elastic-supp orted bistable piezo electric cantilever b eams. Acta Physica Sinica, 2014(09): p. 62-74.

[9] Su, W.J., J. Zu, and Y. Zhu, Design and Development of a Broadband Magnet-induced Dual-cantilever Piezoelectric Energy Harvester. Journal of Intelligent Material Systems \& Structures, 2013. 25(4): p. 430-442.

[10] Eichhorn, C., F. Goldschmidtboeing, and P. Woias, Bidirectional frequency tuning of a piezoelectric energy converter based on a cantilever beam. Journal of Micromechanics \& Microengineering, 2009. 19(9): p. 1693-1696. 